\title{
Efetividade da adesão de sistemas adesivos universais que contém silano em dissilicato de lítio
}

\author{
Effectiveness of universal adhesives systems adhesion that contain silane to lithium \\ disilicate
}

\author{
Andres Felipe Millan Cardenas ${ }^{1}$ Daniella de Oliveira da Silva ${ }^{1}$, Lucas Meneses Lage ${ }^{1}$, Lucila \\ Cristina Rodrigues Araujo', Fabiana Suelen Figuerêdo de Siqueira².
}

\begin{abstract}
Resumo: O objetivo deste trabalho foi avaliar a efetividade de sistemas adesivos universais contendo silano em sua composição na adesão à dissilicato de lítio por meio de uma revisão de literatura. Materiais e Métodos: A seleção de artigos foi realizada através de busca nas bases de dados Pubmed/Medline, LILACS e Web of Science utilizando como descritores/palavras-chave em inglês e português "dissilicato de lítio", "sistemas adesivos universais" e "resistência de união". Após a remoção de duplicatas por título e por resumo, 7 estudos publicados nos últimos 6 anos foram selecionados. Resultados: Foram comparados sistemas adesivos com presença de silano e sistemas adesivos com aplicação de silano separadamente. Foi comprovado que a aplicação do silano promove uma melhor resistência de união ao dissilicato de lítio, mas quando comparados os sistemas adesivos universais contendo silano, á aplicação do silano separadamente melhorou a adesão quando comparado com os sistemas adesivos universais. Conclusão: Os estudos sugerem que os sistemas adesivos universais com silano apresentaram resultados de adesão inferiores quando comparado à técnica de aplicação do silano separadamente no dissilicato de lítio, porém estudos com outros sistemas adesivos universais que contem silano também devem ser realizados.
\end{abstract}

Palavras-chave: dissilicato de lítio; sistemas adesivos universais; resistência de união

\begin{abstract}
A literature review were performed to evaluate the effectiveness of universal adhesive systems that contain silane in the adhesion to lithium disilicate, Materials and Methods: The selection of papers was performed by Pubmed/Medline, LILACS and Web of Science databases using keywords "lithium disilicate", "universal adhesive systems" and "bond strength". After database screening and duplicate removal, 7 studies, published during the last 6 years, were careful examinated. Results: Were compared universal adhesives systems with silane and universal adhesive systems with silane application separately and was corroborated that the silane solution improves the bond strength to lithium dissilicate, However, when compared the silane application separately with universal adhesive contain silane, the application separately increased the adhesion to lithium disilicate. Conclusion: The studies suggest that the universal adhesive systems containing silane result in decreased of adhesion results when compared to silane application separately. However, more studies should be conducted to compare others universal adhesive system that contain silane.
\end{abstract}

Key words: lithium dissilicate; universal adhesive system; bond strength

\footnotetext{
${ }_{1}$ Universidade CEUMA, Programa de Pós-graduação em Odontologia (Maranhão, Brasil)

${ }^{2}$ Centro Universitário Dom Bosco - UNDB, Curso de Odontologia (Maranhão, Brasil)

Autor correspondente: Prof. Dr. Andres Felipe Millan Cardenas. Programa de Pós-Graduação em odontologia. Rua Josué Montello, 1, Renascença II. CEP: 65075-120, São Luís, MA, Brasil. Tel.: (98)98507 4270
} 


\section{Introdução}

Devido a suas ótimas propriedades mecânicas, ópticas, estabilidade e taxa de sobrevivência, as cerâmicas dentais com matriz vítrea tem se mostrado como um dos materiais restauradores indiretos mais utilizados para restabelecer a função, forma e estética dos pacientes 1, 2 . Dentre essas cerâmicas, o dissilicato de lítio destaca-se devido à sua excelente estética, aparência natural, translucidez e alta resistência mecânica 1, 3, 4 .

Embora este tipo de cerâmicas apresentem uma alta resistência à flexão, elas são propensas a fraturas sob carga mastigatória, em virtude de um insatisfatório processo de cimentação. Portanto uma adequada cimentação adesiva deve ser realizada 5 já que é considerado um dos fatores chaves para o sucesso deste tipo de restaurações ${ }^{3}$.

O protocolo de adesão tradicional para o dissilicato de lítio, requer que a superfície interna da cerâmica seja condicionada com ácido fluorídrico (AF) antes da cimentação adesiva ${ }^{6}$. O AF promove a dissolução da fase vítrea da cerâmica e consequentemente aumenta a rugosidade da superfície a ser aderida ${ }^{6-8}$. Após aplicação do AF, um agente de acoplamento silano deve ser aplicado para promover uma adesão bifuncional criando uma interação/absorção química entre a sílica da fase vítrea da cerâmica e os grupos metacrilatos da resina presentes no cimento resinoso através de ligações siloxanas 9, 10, além de incrementar a energia de superfície e molhabilidade da superfície condicionada, diminuindo o ângulo de contato entre a superfície cerâmica e o cimento resinoso ${ }^{11}$.

Mesmo que os fabricantes do silano tenham melhorado este tipo de soluções com a inclusão de monômeros funcionais tais como o 10methacryloyloxydecyl dihydrogen phosphate (10-MDP) para incrementar o potencial de interação química do silano com a superfície cerâmica-dente, a simplificação da técnica clínica faz com que o profissional procure sempre vias mais práticas do que a aplicação separada do silano e do sistema adesivo ${ }^{12}$.

Sistemas adesivos "universais" ou "multi-modo" foram introduzidos no mercado devido a inclusão de monômeros funcionais como o 10-MDP para potencializar a interação química e pela possibilidade de ser utilizados em diferentes substratos como as resinas compostas, dissilicato de lítio, zircônia e ligas metálicas ${ }^{12-14}$.

Outra vantagem de alguns destes sistemas adesivos é a recente introdução do agente de acoplamento silano dentro do sistema adesivo ${ }^{12,15}$. Embora o uso clínico de adesivos universais com silano na mesma solução seja muito conveniente para a adesão no dissilicato de lítio, a mistura entre silano e monômeros resinosos na mesma solução ainda parece controversa, portanto, o objetivo da presente revisão é avaliar a efetividade de sistemas adesivos universais contendo silano na adesão a dissilicato de lítio.

\section{Materiais e métodos}

Foram pesquisados artigos publicados nos últimos 6 anos, nos quais sistemas adesivos universais foram testados na resistência de união à dissilicato de lítio.

As bases de dados utilizadas foram: Pubmed/Medline, LILACS e Web of Science. Os descritores utilizados foram extraídos dos Descritores em Ciências da Saúde (DeCS) em Português e em Inglês Medical Subject Headings (Mesh). Os 
descritores utilizados foram: "Dissilicato de Lítio"; "sistemas adesivos universais"; "resistência de união" para língua portuguesa. Para língua inglesa, foram: "Lithium dissilicate"; "Universal adhesive system"; "Bond Strength".

No Pubmed foram encontrados 8 artigos relacionados, no LILACS 7 artigos foram encontrados e no Web of Science 18 foram encontradas. Um total de 34 artigos foram encontrados. Após a remoção de duplicatas apenas 19 foram mantidos e leituras por título e resumo, apenas 7 foram selecionados $12,15-20$.

\section{Revisão de literatura}

$\mathrm{Na}$ ausência de sistemas adesivos, o agente de acoplamento silano desempenha um papel importante na molhabilidade, uma vez que os cimentos resinosos não podem infiltrar adequadamente nas irregularidades criadas pelo AF para criar uma forte adesão devido a sua viscosidade relativamente alta ${ }^{21}$. A superfície hidrofílica da cerâmica tornase hidrofóbica após a silanização, permitindo que o cimento resinoso molhe de forma ideal a superfície de dissilicato de lítio ${ }^{10,22 .}$

Alguns adesivos universais, tais como 0 Clearfil Universal Bond (Kuraray) e Single Bond Universal (3M), apresentam silano na sua composição. Segundo os fabricantes, a inclusão do silano visa simplificar o protocolo de cimentação das cerâmicas vítreas evitando, teoricamente, a aplicação da solução de silano após 0 condicionamento com AF 23 .

De acordo com ${ }^{15}$, a presença do MDP dentro dos adesivos universais não tem significativa influência nos valores de resistência de união porém, os resultados podem variar de acordo com o tipo de cerâmica de CAD/CAM utilizado.
Entretanto, de acordo com ${ }^{16}$ a resistência de união foi estatisticamente afetada pelo protocolo adesivo utilizado, uma vez que a utilização do agente de acoplamento silano separado promoveu uma mais estável e durável adesão do que quando utilizado sistemas adesivos universais contendo silano na sua composição. Isso revela a importância do agente de acoplamento silano na adesão em cerâmicas de dissilicato de lítio. Estes resultados também foram encontrados por Noda et al ${ }^{17} 2017$. Apesar da presença do silano em alguns sistemas adesivos universais, essa interface adesiva parece ser instável, uma vez que a presença do MDP e água cria um meio ácido e/ou a incorporação de monômeros resinosos pode afetar o desempenho do silano. A presença de monômeros resinosos pode dificultar a evaporação da água que é um subproduto da reação de condensação entre 0 silano e 0 dissilicato de lítio ${ }^{22}$.

Embora estes dois trabalhos anteriores não tenham demostrado uma adequada adesão a dissilicato de lítio quando sistemas adesivos universais foram utilizados 12 , demostraram pelo analise por microRaman, uma interação do sistema adesivo Single Bond Universal após a lavagem da superfície. Segundo os autores, a interação foi observada uma vez que este sistema adesivo é uma mistura balanceada de monômeros de metacrilato, monômero MDP e silano, capaz de promover uma boa adesão imediata quando sistemas adesivos universais foram utilizados.

Assim, é importante destacar que a utilização de sistemas adesivos universais que contem silano em sua composição apresentam resultados de resistência de união inferior ao protocolo padrão, porem superior a sistemas adesivos universais que não contem silano na sua composição ${ }^{12,18}$, 
e isto é mais notório após testes que simulam 0 envelhecimento como termociclagem ou armazenamento em água 12, 16, 18 .

Sabe-se que interfaces adesivas silanizadas expostas a água diminuem a resistência de união devido ao rompimento hidrolítico de ligações siloxano 24, 25. Adicionalmente, os sistemas adesivos também sofrem sorção de água, o que leva a plastificação dos polímeros e consequentemente uma diminuição das propriedades mecânicas ${ }^{26,27}$. Esta significativa diminuição nas propriedades adesivas foi mais observada em estudos quando sistemas adesivos que contem silano na sua composição foram utilizados quando comparados ao silano aplicado separadamente do sistema adesivo ${ }^{12}$, 16, 18 .

Em resumo, a presença do silano dentro do sistema adesivo universal apresentou resultados inferiores em termos de resistência de união quando comparado a aplicação do silano separadamente. No entanto, apenas estudos in vitro suportam estes resultados. Assim, estudos clínicos devem ser conduzidos para avaliar a verdadeira efetividade destes sistemas adesivos universais contendo silano na sua composição.

Adicionalmente, estudos com outros sistemas adesivos universais que contem silano também devem ser realizados, para comparar se o efeito da concentração do silano ou monômero MDP poderiam potencializar a interação com a superfície de dissilicato de lítio.

\section{Conclusão}

Os sistemas adesivos universais que contem silano apresentam resultados de adesão inferior quando comparado a aplicação do silano separadamente. Porém eles apresentam um desempenho superior quando comparados a sistemas adesivos universais sem silano.

\section{Referências}

1. Holand $W$, Schweiger $M$, Frank $M$, Rheinberger $V$. A comparison of the microstructure and properties of the IPS Empress 2 and the IPS Empress glassceramics. J Biomed Mater Res. 2000;53(4):297-303.

2. Collares K, Correa MB, Laske M, Kramer E, Reiss B, Moraes RR, et al. A practice-based research network on the survival of ceramic inlay/onlay restorations. Dent Mater. 2016;32(5):687-94.

3. Tian T, Tsoi JK, Matinlinna JP, Burrow MF. Aspects of bonding between resin luting cements and glass ceramic materials. Dent Mater. 2014;30(7):e147-62.

4. Shibata S, Taguchi C, Gondo R, Stolf SC, Baratieri LN. Ceramic Veneers and DirectComposite Cases of Amelogenesis Imperfecta Rehabilitation. Oper Dent. 2016;41(3):233-42.

5. Dietschi D, Maeder M, Meyer JM, Holz J. In vitro resistance to fracture of porcelain inlays bonded to tooth. Quintessence Int. 1990;21(10):823-31 .

6. Soares CJ, Soares PV, Pereira JC, Fonseca RB. Surface treatment protocols in the cementation process of ceramic and laboratory-processed composite restorations: a literature review. J Esthet Restor Dent. 2005;17(4):224-35.

7. Chen $\mathrm{JH}$, Matsumura $\mathrm{H}$, Atsuta M. Effect of different etching periods on the bond strength of a composite resin to a machinable porcelain. $\mathrm{J}$ Dent. 1998;26(1):53-8.

8. Peutzfeldt A, Sahafi A, Flury S. Bonding of restorative materials to dentin with various luting agents. Oper Dent. 2011;36(3):26673.

9. Lung CY, Matinlinna JP. Aspects of silane coupling agents and surface conditioning in dentistry: an overview. Dent Mater. 2012;28(5):467-77. 
10. Matinlinna JP, Vallittu PK. Bonding of resin composites to etchable ceramic surfaces an insight review of the chemical aspects on surface conditioning. J Oral Rehabil. 2007;34(8):622-30.

11.Phoenix RD, Shen C. Characterization of treated porcelain surfaces via dynamic contact angle analysis. Int $\mathrm{J}$ Prosthodont. 1995;8(2):187-94.

12.Cardenas AM, Siqueira $F$, Hass V, Malaquias $P$, Gutierrez MF, Reis $A$, et al. Effect of MDP-containing Silane and Adhesive Used Alone or in Combination on the Long-term Bond Strength and Chemical Interaction with Lithium Disilicate Ceramics. J Adhes Dent. 2017;19(3):203-12.

13. Hanabusa $M$, Mine A, Kuboki T, Momoi $Y$, Van Ende A, Van Meerbeek B, et al. Bonding effectiveness of a new 'multi-mode' adhesive to enamel and dentine. $J$ Dent. 2012;40(6):475-84.

14.Munoz MA, Luque I, Hass V, Reis A, Loguercio AD, Bombarda $\mathrm{NH}$. Immediate bonding properties of universal adhesives to dentine. J Dent. 2013;41(5):404-11.

15. Siqueira F, Cardenas AM, Gutierrez MF, Malaquias $\mathrm{P}$, Hass $\mathrm{V}$, Reis $\mathrm{A}$, et al. Laboratory Performance of Universal Adhesive Systems for Luting CAD/CAM Restorative Materials. J Adhes Dent. 2016;18(4):331-40.

16. Elsayed A, Younes F, Lehmann F, Kern M. Tensile Bond Strength of So-called Universal Primers and Universal Multimode Adhesives to Zirconia and Lithium Disilicate Ceramics. The journal of adhesive dentistry. 2017;19(3):221-8.

17. Noda Y, Nakajima M, Takahashi M, Mamanee T, Hosaka K, Takagaki T, et al. The effect of five kinds of surface treatment agents on the bond strength to various ceramics with thermocycle aging. Dental Materials Journal. 2017;36(6):755-61.

18.Passia N, Lehmann F, Freitag-Wolf S, Kern $M$. Tensile bond strength of different universal adhesive systems to lithium disilicate ceramic. Journal of the American Dental Association (1939). 2015;146(10):729-34.

19.Perdigao J, Sezinando A, Munoz MA, Luque-Martinez IV, Loguercio AD. Prefabricated veneers - bond strengths and ultramorphological analyses. The journal of adhesive dentistry. 2014;16(2):137-46.
20.Tostes BO. Influência do tempo de condicionamento ácido na alteração superficial, resistência de união e compressão da cerâmica de dissilicato de lítio. 2016:128-.

21.El Zohairy AA, De Gee AJ, Hassan FM, Feilzer AJ. The effect of adhesives with various degrees of hydrophilicity on resin ceramic bond durability. Dent Mater. 2004;20(8):778-87.

22. Chen L, Shen H, Suh BI. Effect of incorporating BisGMA resin on the bonding properties of silane and zirconia primers. J Prosthet Dent. 2013;110(5):402-7.

23.Jorge P, Swift EJ, Jr. Universal Adhesives. Journal of Esthetic and Restorative Dentistry. 2015;27(6):331-4.

24. Matinlinna JP, Lassila LV. Experimental Novel Silane System in Adhesion Promotion between Dental Resin and Pretreated Titanium. Part II: Effect of Long-Term Water Storage. Silicon. 2010;2(2):79-85.

25. Oyague RC, Monticelli F, Toledano M, Osorio E, Ferrari M, Osorio R. Effect of water aging on microtensile bond strength of dualcured resin cements to pre-treated sintered zirconium-oxide ceramics. Dent Mater. 2009;25(3):392-9.

26. Emamieh S, Sadr A, Ghasemi A, Torabzadeh H, Akhavanzanjani V, Tagami $J$. Effects of solvent drying time and water storage on ultimate tensile strength of adhesives. J Investig Clin Dent. 2014;5(1):51-7.

27. Reis $A$, Wambier L, Malaquias $T$, Wambier DS, Loguercio AD. Effects of warm air drying on water sorption, solubility, and adhesive strength of simplified etch-and-rinse adhesives. J Adhes Dent. 2013;15(1):41-6. 